\title{
Diversidade e abundância sazonal da avifauna em duas planícies de maré no estuário da baía da Babitonga, norte de Santa Catarina
}

\author{
Alexandre V. Grose ${ }^{1,3}$, Cristiane C. Hillebrant ${ }^{2,3}$ \& Marta J. Cremer ${ }^{3}$ \\ 1. Programa de Pós-graduação em Zoologia, Universidade Federal do Paraná (UFPR), Av. Cel. Francisco H dos Santos, s/n, Curitiba, PR. (ale.grose@hotmail.com) \\ 2. Rua Leônidas Branco, 219, Centro, 89240-000, São Francisco do Sul, SC. (cris_hillebrand@hotmail.com) \\ 3. Laboratório de Ecologia de Ecossistemas Costeiros, Departamento de Ciências Biológicas, Universidade da Região de Joinville (UNIVILLE), Caixa Postal 110, 89240-000, São Francisco \\ do Sul, SC. (marta.cremer@univille.net)
}

\begin{abstract}
Diversity and abundance of birds in two tidal flat in Babitonga Bay estuary, north of Santa Catarina state, Brazil. Information of birds in estuaries of Santa Catarina is scarce. This work aimed to collect data on diversity, abundance and seasonal variation on this community. Sampling of birds in two tidal flats in Babitonga Bay estuary was carried out during one year (May 2006 to April 2007). A total of 25 species were identified, being 15 in Linguado (LG) and 24 in Monte de Trigo (MT). Only one species was unique in LG (Himantopus melanurus Vieillot, 1817) and 10 in MT. The number of species in MT was higher than in LG due to the conservation condition. The most abundant species on MT was the Black Skimmer [Rynchops niger (Linnaeus, 1758)] and in the LG was the Little Blue Heron [Egretta caerulea (Linnaeus, 1758)]. During some months Nearctic migratory species were recorded in both areas, representing an increase in diversity. The extensive tidal flat formed by the closure of the channel in LG is widely used by birds, possibly because of increased food availability.
\end{abstract}

KEYWORDS. Shorebirds, anthropic areas, Calidris canutus.

RESUMO. Informações sobre a ocorrência de aves nos ambientes estuarinos de Santa Catarina ainda são escassas. O objetivo deste trabalho foi registrar a diversidade, abundância e variação sazonal das aves em duas planícies de maré na baía da Babitonga. As amostragens foram realizadas durante um ano (maio de 2006 a abril 2007). No total foram identificadas 25 espécies, sendo 15 no Linguado (LG) e 24 na desembocadura do Monte de Trigo (MT). Apenas uma espécie foi exclusiva no LG Himantopus melanurus (Vieillot, 1817), enquanto dez espécies ocorreram apenas no MT. O número de espécies em MT foi superior ao encontrado em LG. A espécie mais abundante em MT foi Rynchops niger (Linnaeus, 1758) e em LG foi Egretta caerulea (Linnaeus, 1758). Durante alguns meses foram registradas espécies migratórias neárticas em ambas as áreas, o que representou um acréscimo na diversidade. A extensa planície de maré formada pelo fechamento do canal do Linguado tem sido muito ocupada por aves, possivelmente pela maior disponibilidade de alimento.

PALAVRAS-CHAVE. Aves costeiras, áreas antropizadas, Calidris canutus.

Considerando a extensão costeira e a diversidade de ambientes de transição em Santa Catarina, a informação sobre a avifauna de ambientes estuarinos do estado ainda é escassa, limitando-se a poucos estudos (IBAMA, 1998; BRANCO, 2000; NAKa \& Rodrigues, 2000; PiacentinI \& CAMPBell-Thompson, 2006). As aves são elementos importantes na dinâmica dos ecossistemas onde vivem, o que evidencia sua importância para a conservação (VOOREN \& BRUSque, 1999). Utilizam uma variedade de ambientes formados a partir da transição terra-mar, como baías, estuários, lagunas, planícies de maré e manguezais, entre outros (SCHREIBER \& BURgER, 2002). Durante alguma fase de seu ciclo de vida, diversas espécies de aves concentram-se em estuários e dependem da grande biomassa que há no sedimento das planícies de maré, resultante da produtividade dos ecossistemas costeiros (DiAs et al., 2006).

De acordo com LOURENÇo et al. (2005), planícies de maré são sistemas de alta produtividade, apesar de sua área ser relativamente pequena. Estes ambientes caracterizam-se por apresentar baixa energia devido à reduzida influência de ondas, responsável pela deposição de sedimento arenoso-lodoso (NYBAKKEN, 2001). Em certas épocas do ano sustentam populações de várias espécies de aves, entre elas as migratórias neárticas que fogem do rigoroso inverno da América do Norte (Sick, 1997). Segundo o CEMAVE (2005), 172 espécies de aves migratórias podem ser registradas no
Brasil: 102 (59\%) são oriundas do hemisfério norte e 70 (41\%) provêm do sul do continente, o que representa um acréscimo na diversidade local.

A maré é o principal determinante do período em que as espécies permanecem nessas planícies (ODum, 1997). Nesses ciclos ambientais, o período de exposição do sedimento varia e a movimentação das marés cria breves condições particulares para os animais que habitam o sedimento lodoso e para suas presas. De acordo com Granadeiro et al. (2006), superfícies de sedimentos lodosos nos estuários são áreas indispensáveis para o forrageamento, mas sua exploração fica condicionada aos movimentos de maré e pela salinidade da água intersticial do solo (VOOREN \& BRUSQUe, 1999).

Objetiva-se neste trabalho caracterizar as comunidades de aves associadas a duas áreas de planície de maré, ambas inseridas no estuário da Baía da Babitonga, fornecendo informações sobre a diversidade, abundância e variação sazonal.

\section{MATERIAL E MÉTODOS}

Área de estudo. A baía da Babitonga localizase no litoral norte de Santa Catarina e abrange parte dos municípios de Itapoá, Garuva, Joinville, Araquari, São Francisco do Sul e Barra do Sul (FATMA, 2002). Constitui uma das áreas estuarinas mais importantes 
da costa de Santa Catarina, com uma superfície de manguezais de aproximadamente 6.200 ha. A baía possui águas calmas e quentes, com profundidade média de $6 \mathrm{~m}$ e máxima de 28 m (FATMA, 2002). As margens são formadas por manguezais, praias arenosas e margens rochosas, e em seu interior há cerca de 24 ilhas (IBAMA, 1998). A região apresenta um clima mesotérmico úmido, com temperatura média anual de $20,5^{\circ} \mathrm{C}$ e precipitação média de 2.265 mm (GonçALVES et al., 2006).

Para a realização deste estudo foram amostradas duas áreas de planícies de maré: uma localizada no braço norte do canal do Linguado (LG) $\left(26^{\circ} 21^{\prime} 38,44^{\prime \prime} \mathrm{S}\right.$, $48^{\circ} 39^{\prime} 46,56^{\prime}$ 'O) e a outra na desembocadura do rio Monte de Trigo (MT) $\left(26^{\circ} 12^{\prime} 49,48^{\prime}\right.$ 'S , 48 $\left.35^{\prime} 26,41^{\prime \prime} \mathrm{O}\right)$. O Linguado foi artificialmente fechado em 1907 com o aterro de seus dois braços, mantendo apenas uma abertura de $120 \mathrm{~m}$ no braço sul, sobre a qual foi construída uma ponte metálica. Nesse local, o canal possuía largura de $600 \mathrm{~m}$ e cerca de $2,5 \mathrm{~m}$ de profundidade; o fechamento completo ocorreu em 1939 (CREMER, 2006). Em razão da interrupção do ciclo natural das águas, o assoreamento foi intenso, formando-se extensas planícies de maré, que passaram a oferecer uma área de forrageio para a avifauna da região. Porém, este é um ambiente degradado pela alteração nas condições físico-químicas da água e índices de contaminação (OLIVEIRA et al., 2006), além de ser influenciado diretamente pelo tráfego rodoviário na BR-280. A área amostrada localiza-se na margem oeste do canal.

O rio Monte de Trigo é de médio porte, na porção norte da ilha de São Francisco do Sul. Sua foz, voltada para nordeste, forma ampla coroa de estrutura arenolodosa. A desembocadura do rio está nas proximidades do Saco do Iperoba, uma enseada localizada nas imediações do canal de acesso à baía. Esta região encontra-se próxima à ligação da baía com o Oceano Atlântico e pode ser considerada uma área que sofreu pouca alteração antrópica.

A vegetação de manguezal, característica nesses ambientes, encontra-se nos dois pontos amostrados e a principal espécie em ambas as áreas é Laguncularia racemosa (mangue-branco), embora também ocorram Rizhophora mangle (mangue-vermelho) e Avicennia schaueriana (mangue-preto) (DoRnelles et al., 2006). A vegetação da margem também é composta por plantas herbáceas, como gramíneas do gênero Spartina, que ocupam a transição água/continente.

Coleta e análise de dados. Foram realizados censos quinzenais entre os meses de maio de 2006 e abril de 2007 nas duas planícies de maré selecionadas. Em intervalos regulares de $10 \mathrm{~min}$ foi efetuada a varredura da área para o registro das espécies e do número de indivíduos por espécie; foram contadas todas as espécies que utilizavam a planície de maré, para alimentação e/ ou repouso, assim como aquelas pousadas nas margens. A área de observação compreendeu um ângulo de $180^{\circ} \mathrm{a}$ partir de um ponto fixo na margem e para as amostragens foram utilizados binóculos $(7 \times 50)$. As contagens sempre iniciaram com a maré baixa, considerando as informações obtidas na Tábua de Marés publicada pela Diretoria de Hidrografia e Navegação da Marinha para o Porto de São Francisco do Sul. O período de amostragem foi encerrado quando a planície se encontrava totalmente encoberta pela água, totalizando, em média, cinco horas de observação. A taxonomia e o status de ocorrência das espécies no Brasil foram definidos com base no Comitê Brasileiro de Registros Ornitológicos (CBRO, 2011).

Para avaliação da riqueza foi considerado o número de espécies, analisando os dados por mês e por área. Para a análise da diversidade mensal de cada área foi aplicado o índice de Shannon-Wiener. Considerando que a cada mês foram realizadas duas amostragens por área, foi considerada para o cálculo da abundância mensal apenas aquela que apresentava o maior número de indivíduos de cada espécie (abundância máxima). Para analisar a similaridade entre os meses, foi calculado o índice de similaridade quantitativa de Bray-Curtis, com o modo de agrupamento "Group Average" no programa Primer 5.0 (ClARKE \& GORLEY, 2001).

\section{RESULTADOS}

Foram realizados 47 censos, sendo 24 deles no MT e 23 no LG, totalizando aproximadamente 250 horas de observação. Foram registradas 25 espécies nessas planícies de maré (Tab. I). A família mais representativa foi Scolopacidae, com cinco espécies, compondo 20\% da riqueza, seguida de Ardeidae (quatro espécies; 16\%).

A curva de acumulação de espécies mostrou uma tendência à estabilidade a partir da $12^{\mathrm{a}}$ amostragem no Monte de Trigo, enquanto no Linguado essa tendência foi atingida a partir da $15^{\mathrm{a}}$ amostra (Fig. 1).

$\mathrm{Na}$ planície de maré do MT foram registradas 24 espécies, quase o dobro do encontrado no LG (15 espécies). Entre as espécies identificadas, 14 foram comuns entre as áreas, 10 de ocorrência exclusiva no MT e apenas uma exclusiva no LG.

Em ambas as áreas ocorreram flutuações na riqueza ao longo dos meses. No MT o menor número de espécies foi registrado em abril (8 espécies) e o maior número foi registrado em setembro e outubro (14). No LG o menor e maior número de espécies foi registrado, respectivamente, em maio e novembro (6 espécies) e em janeiro e março (10 espécies) (Fig. 2).

A diversidade manteve-se estável no LG, com um pequeno acréscimo nos meses de verão, quando foi registrado o valor mais alto (janeiro; 1,649), e o menor índice foi verificado no mês de maio $(0,8242)$. Optou-se pela retirada de Rynchops niger (Linnaeus, 1758) para esta análise, registrada somente no MT, devido à grande abundância nos meses de verão, o que poderia distorcer o índice de diversidade durante este período. A variação do índice de diversidade no MT foi superior, sendo que o maior índice ocorreu no mês de agosto $(2,212)$ e o menor em dezembro $(1,443)$ (Fig. 3). Somente no mês de dezembro a diversidade no MT foi inferior à registrada no LG

A abundância média mensal foi de 172 exemplares 
Tab. I. Lista de espécies registradas nas planícies de maré da baía da Babitonga, Santa Catarina, sul do Brasil [R, residente; VN, visitante sazonal oriundo do hemisfério norte (CBRO, 2011); MT, Monte Trigo; LG; Linguado; Min/Max, número mínimo de indivíduos registrado/número máximo de indivíduos registrado; Meses: os números representam os meses em que a espécie foi registrada na região]

\begin{tabular}{|c|c|c|c|c|c|}
\hline Nome Científico & ST & MT & LG & $\operatorname{Min} / \operatorname{Max}$ & Meses \\
\hline \multicolumn{6}{|l|}{ ORDEM PELECANIFORMES } \\
\hline \multicolumn{6}{|l|}{ FAMÍLIA PHALACROCORACIDAE } \\
\hline Phalacrocorax brasilianus (Gmelin, 1789) & $\mathrm{R}$ & $\mathrm{X}$ & $\mathrm{X}$ & $1 / 48$ & $1-12$ \\
\hline \multicolumn{6}{|l|}{ FAMÍLIA ARDEIDAE } \\
\hline Ardea cocoi (Linnaeus, 1766) & $\mathrm{R}$ & $\mathrm{X}$ & $\mathrm{X}$ & $4 / 4$ & $1-12$ \\
\hline Ardea alba (Linnaeus, 1758) & $\mathrm{R}$ & $\mathrm{X}$ & $\mathrm{X}$ & $1 / 12$ & $1-12$ \\
\hline Egretta thula (Molina, 1782) & $\mathrm{R}$ & $\mathrm{X}$ & $\mathrm{X}$ & $1 / 54$ & $1-12$ \\
\hline Egretta caerulea (Linnaeus, 1758) & $\mathrm{R}$ & $\mathrm{X}$ & $\mathrm{X}$ & $1 / 86$ & $1-12$ \\
\hline \multicolumn{6}{|l|}{ FAMÍLIA THRESKIORNITHIDAE } \\
\hline Theristicus caudatus (Boddaert, 1783) & $\mathrm{R}$ & $\mathrm{X}$ & - & $3 / 3$ & 8 \\
\hline Platalea ajaja (Linnaeus, 1758) & $\mathrm{R}$ & $\mathrm{X}$ & $\mathrm{X}$ & $1 / 5$ & $2,3,5,10,12$ \\
\hline \multicolumn{6}{|l|}{ ORDEM ANSERIFORMES } \\
\hline \multicolumn{6}{|l|}{ FAMÍLIA ANATIDAE } \\
\hline Dendrocygna bicolor (Vieillot, 1816) & $\mathrm{R}$ & $\mathrm{X}$ & - & $20 / 20$ & 5 \\
\hline Anas bahamensis (Linnaeus, 1758) & $\mathrm{R}$ & $\mathrm{X}$ & $\mathrm{X}$ & $1 / 7$ & $1,9,12$ \\
\hline Anas sp. & - & $\mathrm{X}$ & - & $2 / 2$ & 3 \\
\hline \multicolumn{6}{|l|}{ ORDEM FALCONIFORMES } \\
\hline \multicolumn{6}{|l|}{ FAMÍLIA FALCONIDAE } \\
\hline Caracara plancus (Miller, 1777) & $\mathrm{R}$ & $\mathrm{X}$ & - & $4 / 4$ & $5,10,11$ \\
\hline \multicolumn{6}{|l|}{ FAMÍLIA RALLIDAE } \\
\hline Aramides cajanea (Statius Muller, 1776) & $\mathrm{R}$ & $\mathrm{X}$ & - & $2 / 2$ & 7,8 \\
\hline \multicolumn{6}{|l|}{ ORDEM CHARADRIIFORMES } \\
\hline \multicolumn{6}{|l|}{ FAMÍLIA CHARADRIIDAE } \\
\hline Charadrius semipalmatus (Bonaparte, 1825) & $\mathrm{VN}$ & $\mathrm{X}$ & $\mathrm{X}$ & $1 / 65$ & $1-3,5,7-12$ \\
\hline Vanellus chilensis (Molina, 1782) & $\mathrm{R}$ & $\mathrm{X}$ & $\mathrm{X}$ & $1 / 5$ & $1-12$ \\
\hline \multicolumn{6}{|l|}{ FAMÍLIA HAEMATOPODIDAE } \\
\hline Haematopus palliatus (Temminck, 1820) & $\mathrm{R}$ & $\mathrm{X}$ & - & $1 / 3$ & 6,9 \\
\hline \multicolumn{6}{|l|}{ FAMÍLIA RECURVIROSTRIDAE } \\
\hline Himantopus melanurus Vieillot, 1817 & $\mathrm{R}$ & - & $\mathrm{X}$ & $1 / 4$ & $1,3,6$ \\
\hline \multicolumn{6}{|l|}{ FAMÍLIA SCOLOPACIDAE } \\
\hline Tringa melanoleuca (Gmelin, 1789) & $\mathrm{VN}$ & $\mathrm{X}$ & - & $1 / 3$ & 5,9 \\
\hline Tringa flavipes (Gmelin, 1789) & $\mathrm{VN}$ & $\mathrm{X}$ & $\mathrm{X}$ & $1 / 44$ & $1,3,7-12$ \\
\hline Numenius phaeopus (Linnaeus, 1758) & $\mathrm{VN}$ & $\mathrm{X}$ & - & $1 / 2$ & 8,9 \\
\hline Calidris canutus (Linnaeus, 1758) & $\mathrm{VN}$ & $\mathrm{X}$ & - & $1 / 1$ & 9 \\
\hline Calidris sp. & - & $\mathrm{X}$ & - & $1 / 1$ & $6-8$ \\
\hline \multicolumn{6}{|l|}{ FAMÍLIA LARIDAE } \\
\hline Larus dominicanus (Lichtenstein, 1823) & $\mathrm{R}$ & $\mathrm{X}$ & $\mathrm{X}$ & $1 / 247$ & $1-12$ \\
\hline \multicolumn{6}{|l|}{ FAMÍLIA RYNCHOPIDAE } \\
\hline Rynchops niger (Linnaeus, 1758) & $\mathrm{R}$ & $\mathrm{X}$ & $\mathrm{X}$ & $1 / 400$ & $1,2,6,10-12$ \\
\hline \multicolumn{6}{|l|}{ ORDEM CORACIIFORMES } \\
\hline \multicolumn{6}{|l|}{ FAMÍLIA ALCEDINIDAE } \\
\hline Megaceryle torquata (Linnaeus, 1766) & $\mathrm{R}$ & $\mathrm{X}$ & $\mathrm{X}$ & $1 / 1$ & $2,10,12$ \\
\hline \multicolumn{6}{|l|}{ ORDEM PICIFORMES } \\
\hline \multicolumn{6}{|l|}{ FAMÍLIA TYRANNIDAE } \\
\hline Pitangus sulphuratus (Linnaeus, 1766) & $\mathrm{R}$ & $\mathrm{X}$ & $\mathrm{X}$ & $1 / 11$ & $1-12$ \\
\hline
\end{tabular}

no MT e de 116 no LG. No LG a espécie mais abundante foi Egretta caerulea (Linnaeus, 1758), representando $45 \%$ da abundância total, com uma média mensal de 52 indivíduos por censo. No MT a espécie mais abundante foi $R$. niger, representando 58,9\% da abundância, com uma média mensal de 101 indivíduos por censo. As duas espécies mais abundantes por área, juntas, representaram $53,4 \%$ da abundância total.

A abundância máxima no MT ocorreu no mês de janeiro e a menor no mês de maio, enquanto que no
LG estes valores foram registrados, respectivamente, em julho e em abril (Fig. 4). Rynchops niger foi responsável pela grande abundância nos meses de verão (dezembro, janeiro e fevereiro) no MT, com o registro de aproximadamente 400 indivíduos em uma única contagem (Fig. 4).

A análise de similaridade quantitativa no LG revelou a formação de dois grandes grupos. Os meses de verão constituíram um agrupamento com mais de $70 \%$ de similaridade, que provavelmente está relacionado à 


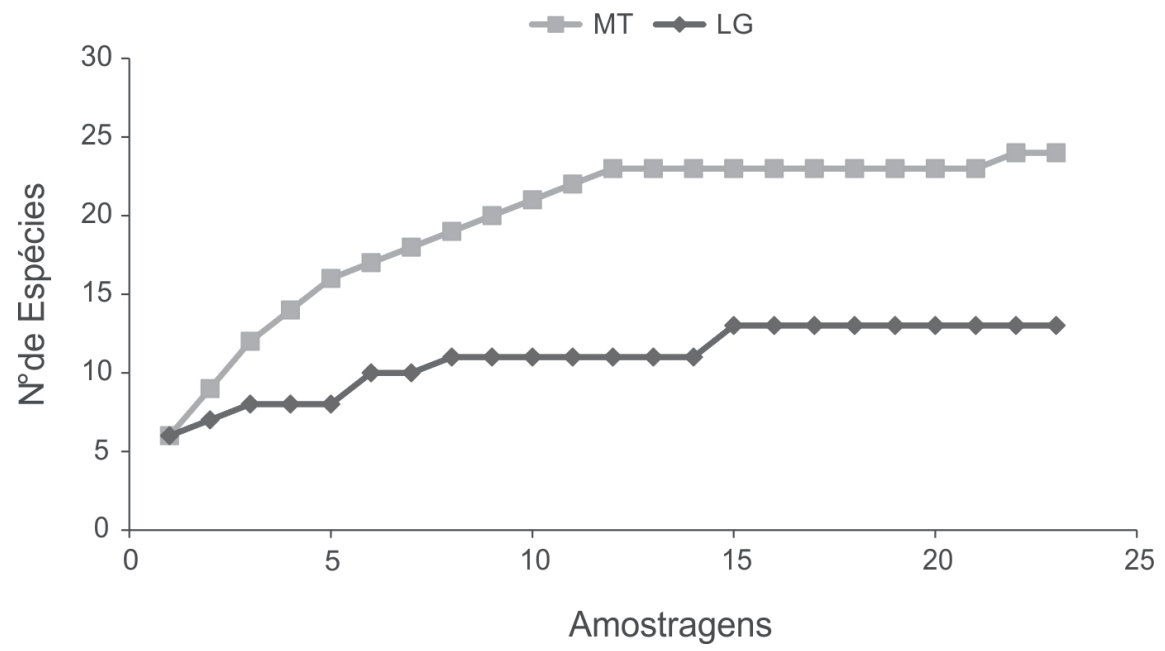

Fig. 1. Curva de acumulação de espécies de aves por área de amostragem em planícies de maré na baía da Babitonga, Santa Catarina, sul do Brasil (LG, Linguado; MT, Monte Trigo).

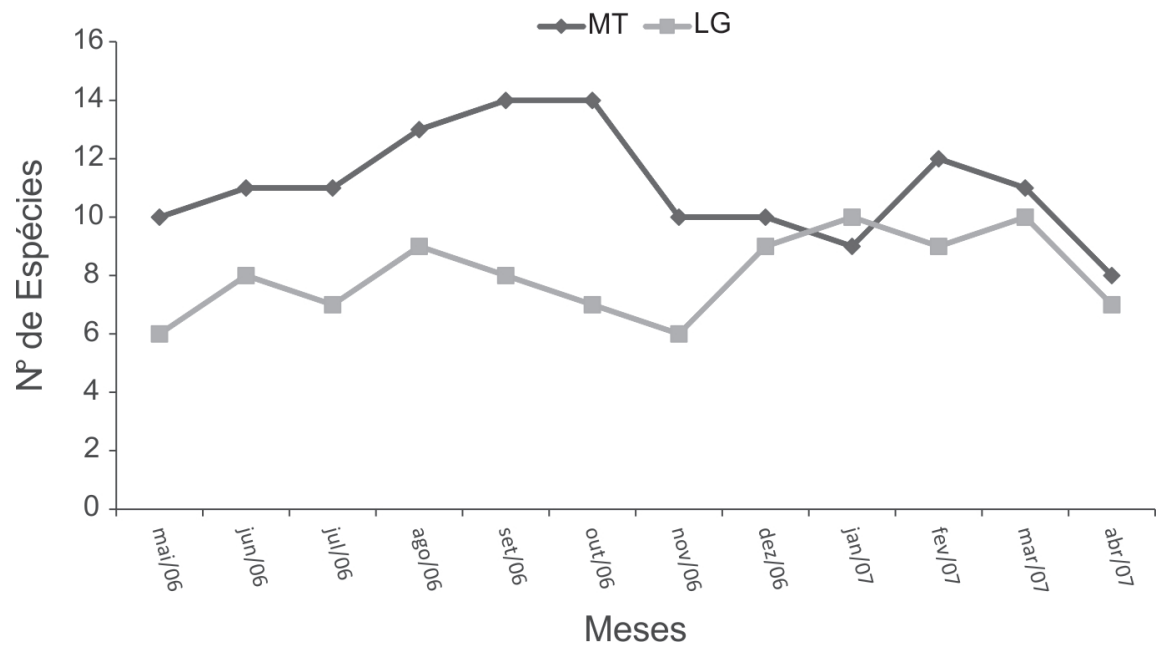

Fig. 2. Variação sazonal na riqueza de espécies de aves por área de amostragem na baía da Babitonga, Santa Catarina, sul do Brasil (LG, Linguado; MT, Monte Trigo).

ocorrência de espécies migratórias neárticas, típicas dessa estação. Os outros meses formaram o outro agrupamento, também com cerca de $70 \%$ de similaridade (maio a novembro), demonstrado pouca variação na composição e estrutura da comunidade ao longo dos meses, com exceção do verão. Apesar de apresentar grande abundância em alguns meses, a diversidade mantevese relativamente baixa. O mês de abril destacou-se dos demais, provavelmente devido à reduzida abundância em relação aos outros meses amostrados (Fig. 5).

Dois agrupamentos também foram formados no MT, mostrando mudanças na comunidade, que se diferenciou em cerca de $55 \%$. Um dos agrupamentos reuniu os meses de primavera e verão (novembro a fevereiro), que revelaram similaridade de cerca de 75\%; nesse período ocorreram as maiores abundâncias. Embora junho tenha sido incluído neste agrupamento, principalmente pela abundância de indivíduos, este mês destacou-se dos demais do grupo em função da diversidade. $\mathrm{O}$ outro agrupamento reuniu os meses de março, abril, julho, agosto, setembro e outubro, mostrando pouca variação na estrutura e composição da comunidade. Maio se diferenciou acentuadamente dos demais, com similaridade de apenas $45 \%$. A abundância neste mês foi a menor em comparação aos outros (Fig. 6).

\section{DISCUSSÃO}

Vários autores descreveram a importância das planícies de maré como fonte de alimento para a comunidade de aves (PIERSMa et al., 1993; Burger et al., 1997; Matsunaga, 2000; De Kam et al., 2004; MaCCARONE \& Brzorad, 2005). Neste trabalho foram registradas 25 espécies que utilizam planícies de maré no estuário, entre espécies tipicamente costeiras, migratórias neárticas, aquáticas e de borda de floresta.

BRANCO (2000), ao estudar a avifauna do estuário do Saco da Fazenda (Itajaí, SC), registrou 45 espécies, das quais 16 também foram detectadas no nosso estudo. Contudo, o índice de diversidade obtido por este autor $(1,75)$ foi um valor intermediário ao calculado para as planícies de maré da baía da Babitonga ( $\mathrm{LG}=1,37$; $\mathrm{MT}=1,86$ ). Dessa forma, pode-se considerar que a avifauna do Saco da Fazenda se caracteriza pela dominância de algumas espécies na comunidade, assim 


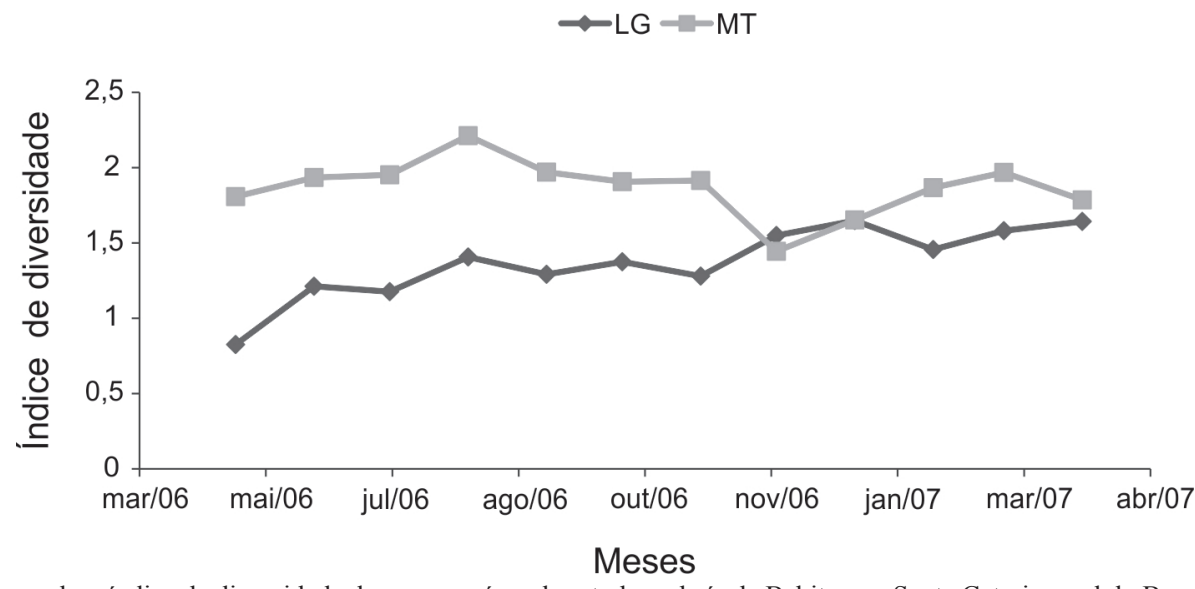

Fig. 3. Variação sazonal no índice de diversidade de aves por área de estudo na baía da Babitonga, Santa Catarina, sul do Brasil, desconsiderando a espécie mais abundante (Rynchops niger) (LG, Linguado; MT, Monte Trigo).

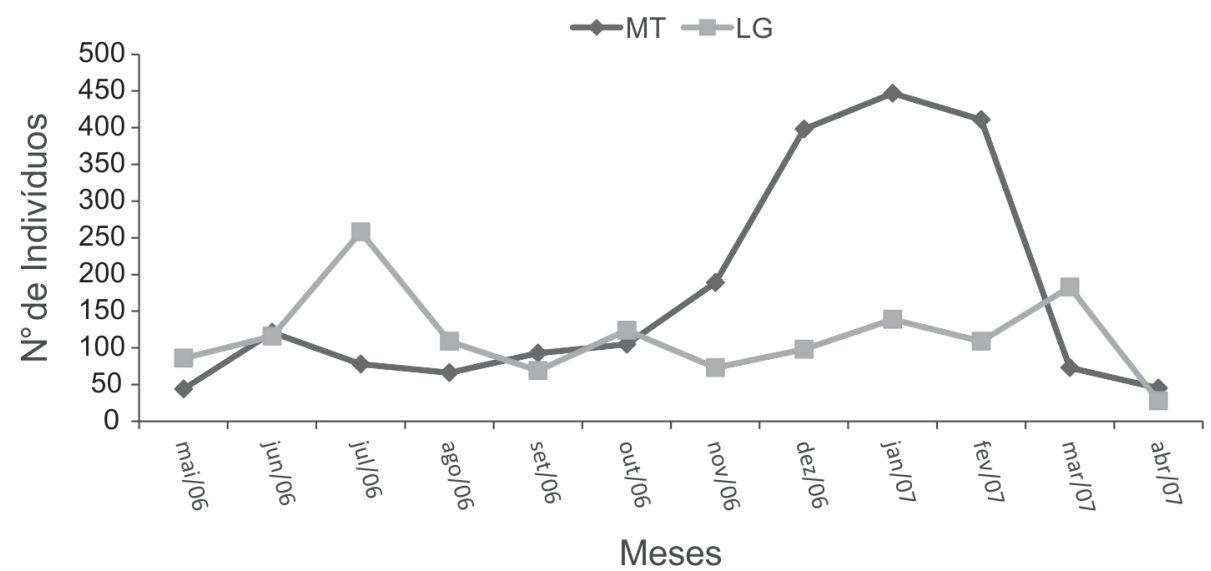

Fig. 4. Variação sazonal na abundância máxima de aves por área de estudo na baía da Babitonga, Santa Catarina, sul do Brasil (LG = Linguado; MT $=$ Monte de Trigo).

como registrado no LG, sendo que ambas constituem áreas com maior influência antrópica. Situação diferente foi verificada na planície do MT, um ambiente mais conservado, onde a diversidade foi relativamente maior.

Algumas famílias podem ser consideradas ocupantes permanentes deste tipo de ambiente, como Ardeidae, Rallidae e Haematopodidae (SICK, 1997) e outras representam um acréscimo na diversidade em alguns meses do ano. A chegada de aves migratórias (AzeVedo-Júnior, 1998; VoOREN \& BRUsque, 1999) como as neárticas (Charadriidae e Scolopacidae) e visitantes (Anatidae e Threskiornithidae), entre outras (SICK, 1997), incrementa a diversidade local.

A maior diversidade registrada na planície do MT pode estar associada à localização da área, que sofre menos influência antrópica e consequentemente favorece a ocorrência de mais espécies sensíveis (BURGER et al., 2004; CESTARI, 2008). Três espécies migratórias neárticas foram exclusivas nessa área: Tringa melanoleuca (Gmelin,1789), Numenius phaeopus (Linnaeus, 1758) e Calidris canutus (Linnaeus, 1758). A planície de maré do Linguado é uma área mais impactada, principalmente pela proximidade da rodovia (BR-280). Neste ambiente foram observadas grandes concentrações de espécies tipicamente tolerantes a ambientes mais alterados, como
Egretta thula (Molina, 1782) e Larus dominicanus (Lichtenstein, 1823) (SICK, 1997; NAKA et al., 2000; BRANCO, 2000).

A elevada abundância no MT nos meses de verão foi associada à chegada de numerosos grupos de $R$. niger à região, que influenciaram consideravelmente o número de indivíduos registrados. Apesar de não se conhecer exatamente seus deslocamentos, VoOREN \& ILHA (1995) confirmaram a chegada de grandes grupos no verão no litoral do Rio Grande do Sul. Branco \& FraCASSO (2005) e Piacentini \& CAmpbell-Thompson (2006) também relataram concentrações da espécie em diversas localidades no litoral de Santa Catarina, com picos nos meses de verão e outono. Outras aves que exibiram maior abundância durante o verão foram Tringa flavipes (Gmelin, 1789) e Charadrius semipalmatus (Bonaparte, 1825), espécies migratórias visitantes da América do Norte (SiCK, 1997; AzEVEdo-JúNIOR, 1998; VoOREN \& BRUSQUE, 1999).

O pico no número de indivíduos registrado em julho para o LG foi fortemente influenciado pela quantidade numerosa de indivíduos de L. dominicanus. Nos meses de inverno inicia o período reprodutivo dessa espécie nas ilhas do arquipélago dos Tamboretes, próximo à costa no litoral de São Francisco do Sul (Branco, 2003). O 

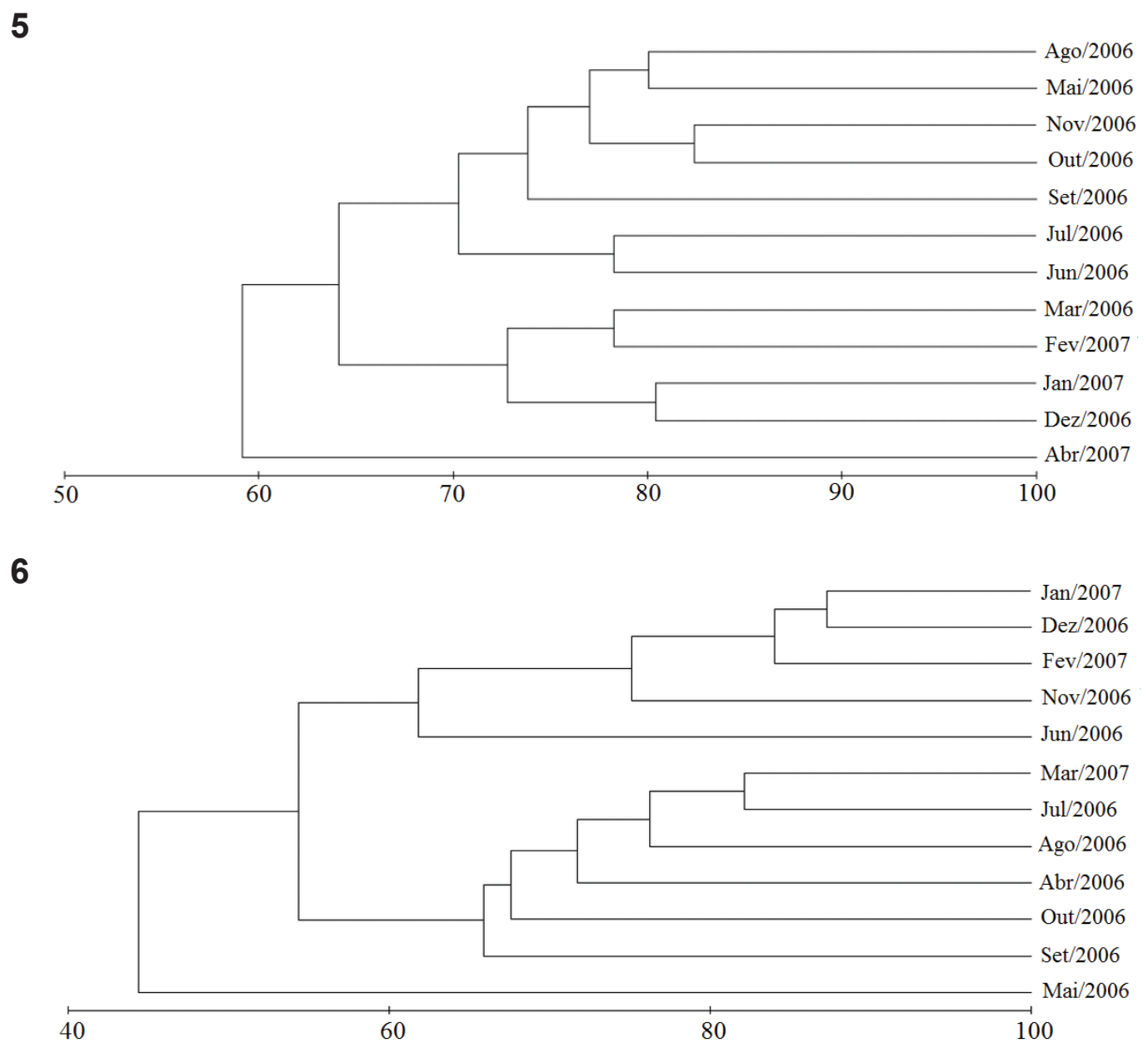

Figs 5, 6. Similaridade quantitativa de Bray-Curtis para as comunidades de aves em planícies de maré ao longo dos meses, baía da Babitonga, Santa Catarina, sul do Brasil: 6, área do Linguado; 7, área do Monte de Trigo.

Linguado, em virtude de sua posição geográfica em relação às ilhas, torna-se um acesso para grandes grupos em direção ao interior do estuário. Possivelmente a concentração registrada esteja associada à maior procura de alimento para suprir as necessidades energéticas no período reprodutivo. Da mesma forma, é possível que a concentração de E. thula e, principalmente, E. caerulea no mês de março esteja associada aos períodos reprodutivos. Ambas iniciam a reprodução no estuário no mês de setembro e a finalizam no mês de março (Alexandre V. Grose, obs. pess.). Essas espécies também apresentam adaptação para alimentação em planícies de maré (Sick, 1997). Nesse contexto, a planície de maré criada artificialmente com o fechamento do Linguado tornou-se uma importante área de alimentação para essas aves.

A observação de algumas espécies em apenas determinados meses do ano, como Anas bahamensis (Linnaeus, 1758), Dendrocygna bicolor (Vieillot, 1816), $R$. niger e Platalea ajaja (Linnaeus, 1758), também foi verificada no manguezal de Santos-Cubatão (SP). São consideradas espécies passageiras, que utilizam a região como ponto de parada, para alimentação e descanso (Olmos \& Silva-e-Silva, 2003).

Cinco espécies migratórias neárticas foram registradas neste estudo: a batuíra-de-bando (C. semipalmatus), o maçarico-de-perna-amarela (T. flavipes), o maçarico-grandede-perna-amarela ( $T$. melanoleuca), o maçarico-de-papovermelho (C. canutus) e o maçarico-galego ( $N$. phaeopus). Elas iniciam sua chegada ao Brasil a partir de agosto, permanecendo até março, fugindo do rigoroso inverno do hemisfério norte à procura de alimento e descanso (SICK, 1997; Vooren \& Brusque, 1999; Telino-JúNIor et al., 2003). Charadrius semipalmatus, T. flavipes e T. melanoleuca já foram observadas em várias localidades de Santa Catarina, como Itajaí (BRANCO, 2000), Ibiraquera (PIACENTINI \& CAMPBell-Thompson, 2006) e Florianópolis (NAKA \& RoDrigues, 2000). Os maçaricos $N$. phaeopus e $C$. canutus ainda não tinham sido avistados no litoral norte e poucos são os registros para Santa Catarina, sendo que o segundo é considerado incomun no estado (RosÁRIO, 1996; NAKA \& RODRIGUES, 2000). A única espécie migratória registrada ao longo de todo o ano foi $C$. semipalmatus, cuja permanência durante os meses de inverno pode estar associada à falta de condicionamento dos jovens e/ou subadultos para o retorno à sua área reprodutiva, devido a acúmulo insuficiente de gordura ou muda incompleta (TeLINO-JÚNIOR et al., 2003). A presença destas espécies reforça a importância da região como parada e descanso durante a migração, dando condições de registro de novas espécies ou ainda pouco conhecidas (GROSE \& CREMER 2011). 
A manutenção das populações de aves na baía da Babitonga pode se tornar comprometida no futuro devido à intensa pressão antrópica registrada na região. Em seu entorno encontra-se o maior centro industrial do estado de Santa Catarina, na cidade de Joinville, e na baía situase o porto de São Francisco do Sul, outro importante polo econômico da região. Vários empreendimentos de grande porte vêm buscando licenciamento e alguns se encontram em implantação. Dessa forma, a Baía da Babitonga vem sofrendo ao longo dos anos sérias ameaças à sua conservação, em um ritmo que reduzirá significativamente suas áreas ainda naturais. Este estudo e sua continuidade devem contribuir principalmente para a seleção de áreas essenciais para a manutenção destas populações de aves, sejam elas residentes ou migratórias, localmente comuns ou ameaçadas de extinção.

Agradecimentos. Os autores agradecem ao Fundo de Apoio à Pesquisa da Universidade da Região de Joinville (FAP/UNIVILLE) pelo apoio logístico e financeiro.

\section{REFERÊNCIAS BIBLIOGRÁFICAS}

Azevedo-Júnior, S. M. 1998. As aves do canal de Santa Cruz, Pernambuco, Brasil. Caderno Ômega da Universidade Federal Rural de Pernambuco, Série Biologia 5:35-50.

Branco, J. O. 2000. Avifauna associada ao estuário do Saco da Fazenda, Itajaí, SC. Revista Brasileira de Zoologia 17(2):387394.

2003. Reprodução das aves marinhas nas ilhas costeiras de Santa Catarina. Revista Brasileira de Zoologia 20(4):619-623.

Branco, J. O. \& Fracasso, H. A. A. 2005. Ocorrência e abundância de Rynchops niger Linnaeus, no litoral de Santa Catarina, Brasil. Revista Brasileira de Zoologia 22(2):430-432.

Burger, J.; Niles, L. \& CLARK, K. E. 1997. Importance of beach, mudflat and marsh habitats to migrant shorebirds on Delaware Bay. Biological Conservation 79(2-3):283-292.

Burger, J.; Jeitner, C.; Clark, K. \& Niles, J. L. 2004. The effect of human activities on migrant shorebirds: successful adaptive management. Environmental Conservation 31(4):283-288.

CBRO - Comitê Brasileiro de Registros Ornitológicos. 2011. Listas das aves do Brasil. Versão 25.01.2011. Disponível em $<\mathrm{http}: / /$ www.cbro.org.br>. Acessado em: 11.03.2011.

CEMAVE - Centro Nacional de Pesquisa para Conservação das Aves Silvestres. 2005. Lista das Espécies de Aves Migratórias Ocorrentes no Brasil. Disponível em: <www.ibama.gov.br/cemave> .

Cestari, C. 2008. O uso de praias arenosas com diferentes concentrações humanas por espécies de aves limícolas (Charadriidae e Scolopacidae) neárticas no sudeste do Brasil Biota Neotropica 8(4)83-88.

Clarke, K. R. \& Gorley, R. N. 2001. PRIMERv5: User Manual/ Tutorial. UK, Primer-E Plymouth. 91p.

Cremer, M. J. 2006. O estuário da baía da Babitonga. In: Cremer, M. J.; Morales, P. R. D. \& Oliveira, T. M. N. eds. Diagnóstico ambiental da Baía da Babitonga. Joinville, Editora Univille, p.15-19.

De-Kam, V. J.; Ens, B.; Piersma, T. \& Zwarts., L. 2004. Shorebirds: an illustrated behavioural ecology. Utrecht, KNNV Publishers. $368 \mathrm{p}$

Dias, M. P.; Granadero, J. P.; Martins R. C. \& Palmeirim, M. J. 2006. Estimating the use of tidal flats by waders: inaccuracies due to the response of birds to the tidal cycle. Bird Study 53(1):32-38.

Dornelles, S. S.; Moreira, G. M. \& Freitas, L. M. 2006. Caracterização da estrutura vegetal dos manguezais do Canal do Linguado, Baía da Babitonga. In: Cremer, M. J.; Morales, P. R.
D. \& Oliveira, T. M. N. eds. Diagnóstico ambiental da Baía da Babitonga. Joinville, Editora Univille, p.187-199.

FATMA - Fundação do Meio Ambiente de Santa Catarina. 2002. Atlas Ambiental da Região de Joinville: Complexo Hídrico da Baía da Babitonga. Florianópolis, Fatma. 144p.

Gonçalves, M. L.; Zanotelli, C. T. \& Oliveira, F. A. 2006. Diagnóstico e prognóstico das disponibilidades e demandas hídricas do rio Cubatão do Norte - Joinville - Santa Catarina Joinville, Editora Univille. 96p.

Granadeiro, J. P.; Dias, M. P.; Martins, R. C. \& Palmeirim, J. M. 2006. Variation in numbers and behavior of waders during the tidal cycle: implications for the use of estuarine sediment flats Acta Oecologica 29(3):293-300.

Grose, A. V. \& Cremer, M. J. 2011. Novo registro do falaropo-debico-grosso Phalaropus fulicarius (Aves, Charadriiformes) no Brasil. Ararajuba 19(4):552-553.

IBAMA - Instituto Brasileiro do Meio Ambiente e dos Recursos Naturais Renováveis 1998. Proteção e controle de ecossistemas costeiros: manguezal da Baía de Babitonga. Brasília, Ibama. $146 \mathrm{p}$.

Lourenço, P. M.; Granadeiro, J. P. \& Palmeirim, J. M. 2005 Importance of drainage channels for waders foraging on tidal flats: relevance for the management of estuarine wetlands. Journal of Applied Ecology 42(3):447-486.

Maccarone, A. D. \& Brzorad, J. N. 2005. Foraging microhabitat selection by wading birds in a tidal estuary, with implications for conservation. Waterbirds 28(3):383-391.

Matsunaga, K. 2000. Effects of tidal cycle on the feeding activity and behavior of Grey Herons in a tidal flat in Notsuke Bay, Northern Japan. Waterbirds 23(2):226-235.

Naka, L. N. \& Rodrigues, M. 2000. As aves da Ilha de Santa Catarina. Florianópolis, UFSC. 294p.

Naka, L. N.; Barnett, J. M.; Kirwan, G.; Tobias, G. \& Azevedo, M A. G. 2000. New and noteworthy bird records from Santa Catarina state. Bulletin of the British Ornithologists Club 120(4):237250.

NYBAKKEN, J. W. 2001. Marine biology: an ecological approach. San Francisco, Benjamin Cummings. 516p.

Odum, E.P. 1997. Fundamentos de Ecologia. Lisboa, Fundação Calouste Gulbenkian. 927p.

Oliveira, T. M. N.; Tureck, C. R.; Bassfeld, J. C.; Torrens, B. M. O.; FARIA, J. M. \& BrasiL, K. 2006. Integridade Ambiental da Baía da Babitonga: Características Físico-químicas, Microbiológicas e Ecotoxicidade. In: Cremer, M. J.; Morales, P. R. D. \& Oliveira, T. M. N. eds. Diagnóstico ambiental da Baía da Babitonga Joinville, Editora da Univille, p. 20-80.

Olmos, F. \& Silva-E-Silva, R. 2003. Guará: ambiente, flora e fauna dos manguezais de Santos-Cubatão. São Paulo, Empresa das Artes. 216p.

Piacentini, V. Q. \& Campbell-Thompson, E. R. 2006. Lista comentada da avifauna da microbacia hidrográfica da Lagoa de Ibiraquera, Imbituba, SC. Biotemas 19(2):55-65.

Piersma, T.; Goeis, P. \& Tulp, I. 1993. An evaluation of intertidal feeding habitats from a shorebird perspective: towards relevant comparisons between temperate and tropical mudflats. Netherlands Journal of Sea Research 31(4):503-512.

RosÁrio, L. A. 1996. As aves em Santa Catarina: distribuição geográfica e meio ambiente. Florianópolis, Fatma. 326p.

SCHREIBER, E. A. \& Burger, J. 2002. Biology of marine birds. Boca Raton, CRC Press. 722 p.

Sick, H. 1997. Ornitologia brasileira. Rio de Janeiro, Nova Fronteira. 862 p.

Telino-Júnior, W. R.; Azevedo-Júnior, S. M. \& Neves, R. M. L. 2003. Censo de aves migratórias (Charadriidae, Scolopacidae e Laridae) na Coroa do Avião, Igarassu, Pernambuco, Brasil. Revista Brasileira de Zoologia 20(3):451-456.

Vooren, C. M. \& Brusque, L. F. 1999. As aves do Ambiente Costeiro do Brasil: Biodiversidade e Conservação. Rio de Janeiro, FUNBIO. 58p.

Vooren, C. \& IlHa, H. H. 1995. Guia das aves comuns da costa do Rio Grande do Sul. Imago Maris 2(1):1-23.

Recebido em 25 de outubro de 2010. Aceito em 29 de março de 2013. ISSN 0073-4721

Artigo disponível em: www.scielo.br/isz 